\title{
Carbon-Enhanced, Metal-Poor Stars and Modeling of the Asymptotic Giant Branch
}

\author{
Sara Lucatello $^{\mathrm{A}, \mathrm{B}, \mathrm{D}}$, Thomas Masseron ${ }^{\mathrm{C}}$, and Jennifer A. Johnson ${ }^{\mathrm{C}}$ \\ A Excellence Cluster Universe, Technische Universität München, Boltzmannstr. 2, \\ D-85748, Garching, Germany \\ B INAF-Osservatorio Astronomico di Padova, vicolo dell'Osservatorio 5, 35122, Padova, Italy \\ ${ }^{C}$ Department of Astronomy, Ohio State University, 140 W. 18th Ave., Columbus, OH 43210, USA \\ D Corresponding author. Email: sara.lucatello@universe-cluster.de
}

\section{Received 2008 December 16, accepted 2009 July 31}

\begin{abstract}
The very high fraction of C-enriched stars among the low end of the Galactic-halo metallicity distribution, along with the wide variety in content of their neutron-capture elements, make the understanding of their formation scenario(s) of extreme interest. The combination of accurate determinations of their chemical composition with long-term radial-velocity monitoring is crucial to address this issue. We discuss the first results of long-term radial-velocity monitoring for $\mathrm{C}$-enhanced, metal-poor stars and explore the connection with chemical peculiarities; the data suggest that belonging to a close binary affects nucleosynthesis on the Asymptotic Giant Branch. We also present abundances for the poorly studied element fluorine in a sample of $10 \mathrm{C}$-enhanced stars with metallicities ranging from $[\mathrm{Fe} / \mathrm{H}]=-1.3$ to -2.9 and compare our measurements to the predictions of state-of-the-art nucleosynthesis models. We show how the observed abundances of fluorine in CEMP stars are considerable lower than expected on the basis of the current state-of-the-art theoretical calculations, hinting at our poor understanding of the nucleosynthetic processes that produce this element.
\end{abstract}

Keywords: binaries: general — stars: AGB and post AGB — stars: carbon

\section{Introduction}

Carbon stars have been recognized as a class of astronomical objects for over a century. They have been distinguished between intrinsic and extrinsic. In the former case, the $\mathrm{C}$ comes from internal origin, such as in the case of Asymptotic Giant Branch (AGB) stars; in the latter case, the $\mathrm{C}$ overabundance is due to mass accretion from a low mass AGB companion $\left(1.5<M / M_{\odot}<3\right)$, which is now a faint and unobservable white dwarf. Examples of extrinsic C-rich objects are classical $\mathrm{CH}$ stars and $\mathrm{Ba}$ stars (McClure 1984a,b). These objects make up a few percent of solar metallicity and mildly metal poor stars $(-1.5<[\mathrm{Fe} / \mathrm{H}]<0)^{1}$.

In the last 15 years, the past and ongoing searches for extremely metal poor stars in the Galaxy have been finding an unexpectedly high number of C-rich stars among the most metal poor objects, the so-called C-enhanced, metal poor stars (CEMP). Although quantitative estimates for their incidence vary depending on the sources, ranging from 9\% (Frebel et al. 2006) to 21\% (Lucatello et al. 2006), there is complete consensus on such fraction being significantly higher than at solar metallicity. This phenomenon is clearly illustrated in Figure 1 (Beers 2006), which shows $[\mathrm{C} / \mathrm{Fe}]$ as a function of $[\mathrm{Fe} / \mathrm{H}]$ for a sample of over 5000 stars observed by the SEGUE collaboration

\footnotetext{
${ }^{1}$ Hereafter $\log \epsilon(A)=\log N(A)+12,[\mathrm{X} / \mathrm{Y}]=\log \left(X / X_{\odot}\right)-\log \left(Y / Y_{\odot}\right)$.
}

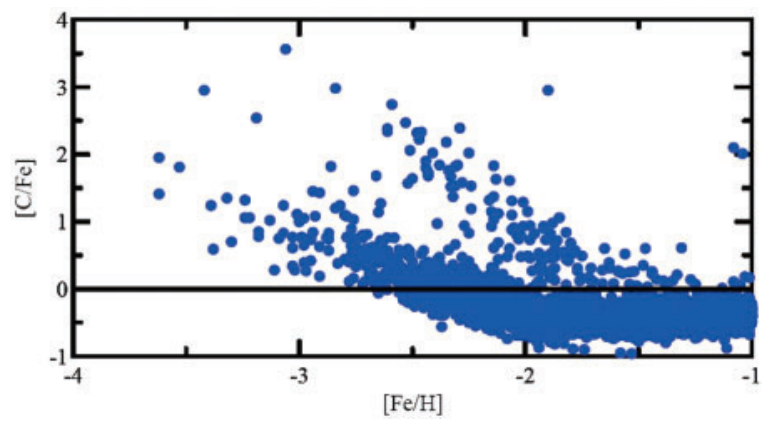

Figure 1 A plot of [C/Fe] as a function of $[\mathrm{Fe} / \mathrm{H}]$ for a sample of over 5000 stars from the SEGUE survey (from Beers 2006).

(Newberg \& Sloan Digital Sky Survey Collaboration 2003).

\section{Types of CEMP Stars}

While all the CEMP stars are characterized by various degrees of $\mathrm{C}$ and $\mathrm{N}$ overabundances, at least three different subclasses emerge when considering the overall abundance pattern, and in particular that of the heavy elements.

By far, the most numerous class ( 70\% of CEMP; Aoki et al. 2007) is that of CEMP that show an enhancement in slow neutron-capture (hereafter, $s$-process) elements (CEMPs stars). It has been shown that long term radial velocity data is consistent with them being all members of double systems, therefore, much like classical $\mathrm{CH}$ and $\mathrm{Ba}$ 
stars (McClure 1984a,b), they owe their peculiar compositions to mass transfer from a now extinct, AGB companion.

About a third (Aoki et al. 2007) of CEMP stars (CEMPno) have no enhancements in neutron-capture elements. Three hypotheses have been put forward for their formation.

Ryan et al. (2005) suggested that CEMPno stars are born from $\mathrm{C}$-enhanced gas enriched by a previous generation of stars. Some of the $\mathrm{C}$ is then turned into $\mathrm{N}$ during the evolution of the present stars. This scenario however fails to explain the existence of dwarf CEMPno objects which are observed (Masseron et al. 2009; S. Lucatello, R. Gratton, T. Masseron, J. A. Johnson, F. Herwig and S. Tsangarides, unpubl. data).

Another possibility is that of self enrichment. Because of their extremely low metal content the stars undergo mixing processes leading them to be enriched in $\mathrm{C}$ and $\mathrm{N}$ at a much lower masses than at solar or slightly subsolar metallicity (Fujimoto, Ikeda \& Iben 2000, case I and II). In principle, this scenario can account for CEMPno peculiar composition. However, such scenarios still cannot explain the existence of dwarf CEMPno (at the earliest such enrichment can happen at the tip of the giant branch). Besides, although CEMPno seem to have an upper metallicity cutoff, most of the objects known so far are well above the $[\mathrm{Fe} / \mathrm{H}]<-3.0$ quoted by Fujimoto et al. as the upper metallicity limit for the onset of the peculiar mixing processes discussed.

The third suggested scenario is the mass transfer from a more massive companion early in its AGB phase, before any considerable $s$ process takes place could fully explain the observed abundance pattern. This scenario would imply that all CEMPno stars are members of binary system (Masseron et al. 2009). However there are issues with this scenario, e.g. that such a companion is expected to produce substantially more $\mathrm{N}$ than $\mathrm{C}$, making the stars essentially $\mathrm{N}$ rather than $\mathrm{C}$ rich. Unfortunately, radial-velocity measurements with a wide enough temporal baseline are available only for a small number of these stars, therefore it is yet not possible to establish whether binarity is a key element in the enrichment scenario.

Finally, only one known star (see e.g. Sneden et al. 2003) shows, along with high $\mathrm{C}$ and $\mathrm{N}$ abundance, a high enhancement in rapid neutron-capture ( $r$-process hereafter) elements (CEMPr stars), the star shows radial velocity variations (Preston \& Sneden 2001), however it is impossible from just a single object to draw any conclusion on the relationship between binarity and chemical composition.

\section{CEMPs Stars and AGB Models}

Given their proposed formation scenario, the surface composition of CEMPs stars bears the imprint of the nucleosynthesis that took place in its companion, the formerly AGB star, which is now a faint and unobservable white dwarf. Therefore the observation of CEMPs stars gives us direct insight into a population of stars that does not exist anymore, offering the ideal testing bench for low metallicity AGB models (even it should be kept in mind some degree of dilution/mixing due to the first dredgeup and/or possibly thermohaline mixing, e.g. Stancliffe et al. 2007, might affect some or all the abundances of the accreted species in the observed stars).

In the last few years, a growing number of high resolutions spectroscopic analyses of CEMPs stars has become available in the literature. As predicted by AGB models (see e.g. Gallino et al. 1998), their compositions are characterized by extreme $s$-process patterns, where, because of their low metallicity, the high number of neutrons available per Fe seed leads the bulk of nucleosynthesis products to shift first past the $\mathrm{Zr}$ peak and then past the Ba peak, accumulating at the termination point of the $s$-process, i.e. essentially on $\mathrm{Pb}$ (and $\mathrm{Bi}$ ).

Current low-mass $\left(1.5<M / M_{\odot}<3\right)$, low-metallicity AGB shell nucleosynthesis models generally predict satisfactorily the observed abundances from $\mathrm{C}$ to $\mathrm{Pb}$ (see e.g. Aoki et al. 2000; Lucatello et al. 2003; Gallino et al. 2005; Sivarani et al. 2006) with two noteworthy exceptions: N and Eu. Nitrogen abundance is consistently found to be very high in CEMPs stars, usually just slightly lower than that of carbon, whereas the theoretical prediction is of a negligible amount of $\mathrm{N}$ production. The reason for this discrepancy is probably to be looked for in the current lack of the inclusion of some degree of Cool Bottom Processing (Nollett, Busso \& Wasserburg 2003).

Most CEMPs stars show an abundance of Europium which is larger than model predictions, in particular the measured $[\mathrm{Eu} / \mathrm{Ba}]$ ratio is generally considerably higher than that resulting from $s$-process nucleosynthesis calculations.

There is currently no explanation for the origin of such discrepancy. Current models allow very little room in the change of the $[\mathrm{Eu} / \mathrm{Ba}]$ ratio, reaching at most $\sim-0.6$ (see e.g. Cristallo et al. 2007).

Eu abundance is measured from just a handful of lines, often from just one, the Eu II line at $\sim 4129 \AA$, the strongest unblended one in the visible spectrum. While blending with a non accounted for $\mathrm{CH}$ or unidentified feature(s) is possible in principle, it seems highly unlikely to be the cause of such large discrepancy. In fact, whenever more than one line is available the abundances derived from different lines are very consistent with each other. Moreover, the lines used to measure $\mathrm{Eu}$ abundances in these objects are characterized by complex profiles due to their hyperfine splitting, which is very well reproduced by just the Eu lines themselves (with appropriate hyperfine splitting inclusion), showing no residual that can be attributed to an additional feature. Finally, when evaluating the $[\mathrm{Eu} / \mathrm{Ba}]$ ratio, both species are measured in their first ionized state, minimizing the effect of uncertainties in the atmospheric parameters. Summarizing, while a modest error in the measured $[\mathrm{Eu} / \mathrm{Ba}]$ ratio is definitely possible, a decrease in the measurement comparable to that needed to match the theoretical predictions, can be definitely ruled out.

Because of the impossibility of explaining the data on the basis of the state-of-the-art $s$-process nucleosynthesis 
models, it has been postulated that the Eu overabundance is due to a separate nucleosynthetic process, an $r$-process enrichment, so that the CEMPs stars with $[\mathrm{Eu} / \mathrm{Ba}]$ ratios higher with respect to that predicted by the models have been classified by some authors as CEMPr/s stars (Beers \& Christlieb 2005).

Except for their overabundances in Eu, the CEMPr/s stars do not show systematic differences from the CEMPs: their metallicity, $\mathrm{C}, \mathrm{N}, \mathrm{Ba}$ distributions are very similar as are their binary fractions (S. Lucatello, R. Gratton, T. Masseron, J. A. Johnson, F. Herwig and S. Tsangarides, unpubl. data).

Several scenarios have been proposed to explain the abundance patterns observed in CEMPr/s:

\subsection{Independent Enrichment Sources}

To account for the abundance pattern observed in CS 29497-030, Ivans et al. (2005) propose a scenario involving a binary star formed from $r$-process, pre-enriched gas and a subsequent $s$-process enrichment due to mass transfer from the companion in its AGB stage. While this scenario can explain a few particular cases such as this one, the very high incidence of CEMPr/s among CEMP and with respect to CEMPs, suggest that this cannot explain the bulk of CEMPr/s. In fact, if the two processes ( $r$ - and $s$ enrichment) were independent, we would expect to find a similar fraction of $r$-enrichment among stars not enhances in $\mathrm{C}$ and $s$-process elements, which is not the case (see Barklem et al. 2005).

\subsection{AGB and SN Type 1.5}

Zijlstra (2004) proposed that CEMPr/s stars are members of binaries with intermediate mass star (IMS) companions (3-8 $\mathrm{M}_{\odot}$ ) which after their AGB phase explodes as a SN type 1.5 that produces $r$-process elements. To reach sufficient core growth to produce a SN type 1.5 from this mass range, very low mass loss and very little dredge-up are needed. This has been shown to be possible in metal free stars (Lau, Stancliffe \& Tout 2008). This scenario, however, fails to reproduce the observed pattern, as AGB stars in that mass range are very inefficient in the production of $s$-process elements (see Busso et al. 2001 and references therein). Moreover, because HBB is expected to happen in IMS, this scenario predicts $\mathrm{N}$ abundances (much) larger than the $\mathrm{C}$ ones, which is at variance with observations. Similar considerations apply to the scenario proposed by Wanajo (2006).

\subsection{AGB and Accretion-Induced Collapse (AIC)}

Another proposed scenario is that involving a binary with a low mass $\left(M<4 \mathrm{M}_{\odot}\right)$ primary which dumps $s$-process enriched material on the observed star during its AGB phase. Later, when the secondary evolves, reverse mass transfer onto the white dwarf takes place, triggering the AIC, and the subsequent neutrino wind, leading to $r$-process elements production (see e.g. Zhang et al. 2006). Given the requirement for reverse mass transfer, in this scenario, all CEMPr/s should be evolved stars. This is not the case, as there are several unevolved CEMPr/s stars (see e.g. Ivans et al. 2005; Cohen et al. 2006), ruling out this possibility.

\subsection{AGB Mass Transfer Only}

Johnson \& Bolte (2004) proposed that the abundance patterns observed in CEMPr/s arise simply from AGB transfer and that the current models for $s$-process nucleosynthesis at low $Z$ are incomplete and that some other 'extra' $s$ process is at work. Masseron \& Johnson (2008) found correlations between $\mathrm{N}$ and $s$-process abundances and suggest that the ${ }^{22} \mathrm{Ne}(\alpha, n)^{25} \mathrm{Mg}$ neutron source dominates in CEMPr/s stars unlike in CEMPs stars where ${ }^{13} \mathrm{C}(\alpha, n){ }^{16} \mathrm{O}$ is the dominant one. However, if indeed the ${ }^{22} \mathrm{Ne}$ source were the dominant one a correlation between $\mathrm{C}+\mathrm{N}+\mathrm{O}$ and the $s$-process enhancement should be expected ( $\mathrm{N}$ abundances at the surface and in the intershell — that becomes ${ }^{22} \mathrm{Ne}$ by double $\alpha$ capture - are different and the latter should be much higher: the intershell material will be made up of the ashes of CNO-cycled material in which most of the CNO elements will have been processed to nitrogen, and not just the nitrogen as seen at the surface).

In order to shed light on this issue and probe these scenarios, information about binarity membership and orbital parameters is of great importance. A few years ago, we started a long term radial velocity monitoring of CEMP stars. Several high-precision radial-velocity measurements for a sample of 47 CEMP stars, all of which have accurate abundance determinations, were collected with FEROS at ESO 2.2-m, SARG at TNG and Hamspec at Lick Obsrevatory, over the span of over four years. Our measurements were combined with similar precision determinations already present in the literature.

The survey is still ongoing, however so far we could determine (in some cases re-determine) accurate orbital periods for seven CEMP stars. This increases by more than a factor of three the number of CEMP with well determined orbital period. Table 1 lists all the CEMP stars with well determined orbital elements, their orbital periods, Fe, $\mathrm{C}, \mathrm{N}, \mathrm{Ba}, \mathrm{Eu}$ and $\mathrm{Pb}$ abundances with appropriate references for them and for the radial velocity used to determine the orbital parameters.

Full details about the survey, observations and the techniques adopted will be given in an upcoming paper (Lucatello et al. in preparation), we here present an intriguing finding that emerged from our first results. Figures 2, 3 and 4 plot the different abundance ratios as a function of the logarithm of the orbital period and show that there is a relationship between the orbital period and the abundance ratios observed on their surface. It is important to keep in mind that objects with a very short period ( $P \leq 100$ days $)$ have likely undergone Roche-Lobe overflow and probably went through a common envelope phase. It is noteworthy how the objects with a short period have more extreme abundance patterns. In the case of $[\mathrm{C}+\mathrm{N} / \mathrm{Fe}]$ such a relationship could in principle be explained by a higher mass transfer efficiency in a close rather than in a loose binary. 
Table 1. CEMP stars with well-determined orbital parameters

\begin{tabular}{lcccccccl}
\hline Star ID & $P($ days $)$ & {$[\mathrm{Fe} / \mathrm{H}]$} & {$[\mathrm{C} / \mathrm{Fe}]$} & {$[\mathrm{N} / \mathrm{Fe}]$} & {$[\mathrm{Ba} / \mathrm{Fe}]$} & {$[\mathrm{Eu} / \mathrm{Fe}]$} & {$[\mathrm{Pb} / \mathrm{Fe}]$} & $\mathrm{RV}$ sources \\
\hline HE0024-2523 & $3.41^{\mathrm{a}}$ & $-2.73^{\mathrm{a}}$ & $2.60^{\mathrm{a}}$ & $2.10^{\mathrm{a}}$ & $1.40^{\mathrm{a}}$ & $<1.10^{\mathrm{a}}$ & $3.4^{\mathrm{a}}$ & $\mathrm{a}$ \\
CS29497-30 & $342^{\mathrm{c}}$ & $-2.57^{\mathrm{b}}$ & $2.47^{\mathrm{b}}$ & 2.12 & 2.32 & $1.99^{\mathrm{b}}$ & $3.65^{\mathrm{b}}$ & $\mathrm{c}, 1$ \\
CS22948-27 & $425.1^{\mathrm{g}}$ & $-2.48^{\mathrm{f}}$ & $2.43^{\mathrm{f}}$ & $1.75^{\mathrm{f}}$ & $2.26^{\mathrm{f}}$ & $1.88^{\mathrm{f}}$ & $2.72^{\mathrm{f}}$ & $\mathrm{d}, \mathrm{e}, \mathrm{f}, \mathrm{g}$ \\
CS22956-28 & $1312^{\mathrm{g}}$ & $-2.05^{\mathrm{c}}$ & $1.74^{\mathrm{c}}$ & $1.50^{\mathrm{g}}$ & $0.60^{\mathrm{g}}$ & $0.8^{\mathrm{g}}$ & $1.90^{\mathrm{g}}$ & $\mathrm{c}, \mathrm{d}, \mathrm{g}$ \\
CS22942-19 & $3616^{\mathrm{g}}$ & $-2.64^{\mathrm{h}}$ & $2.00^{\mathrm{h}}$ & $0.80^{\mathrm{h}}$ & $1.92^{\mathrm{h}}$ & $<0.79^{\mathrm{h}}$ & $1.6^{\mathrm{h}}$ & $\mathrm{d}, \mathrm{h}$ \\
LP625-44 & $4547^{\mathrm{g}}$ & $-2.72^{\mathrm{h}}$ & $2.25^{\mathrm{h}}$ & $0.95^{\mathrm{h}}$ & $2.81^{\mathrm{h}}$ & $1.72^{\mathrm{h}}$ & $2.60^{\mathrm{h}}$ & $\mathrm{g}, \mathrm{h}, \mathrm{i}, \mathrm{j}$ \\
CS29497-34 & $471^{\mathrm{g}}$ & $-2.90^{\mathrm{f}}$ & $2.63^{\mathrm{f}}$ & $2.38^{\mathrm{f}}$ & $2.03^{\mathrm{f}}$ & $1.80^{\mathrm{f}}$ & $2.95^{\mathrm{f}}$ & $\mathrm{e}, \mathrm{f}, \mathrm{g}$ \\
CS22957-27 & $1081^{\mathrm{g}}$ & $-3.12^{\mathrm{m}}$ & $2.14^{\mathrm{m}}$ & $1.89^{\mathrm{m}}$ & $-0.78^{\mathrm{m}}$ & & & $\mathrm{d}, \mathrm{g}, \mathrm{h}, \mathrm{m}$ \\
LP706-7 & $1130^{\mathrm{g}}$ & $-2.74^{\mathrm{h}}$ & $2.15^{\mathrm{h}}$ & $1.80^{\mathrm{h}}$ & $2.01^{\mathrm{h}}$ & $1.40^{\mathrm{h}}$ & $2.28^{\mathrm{h}}$ & $\mathrm{g}, \mathrm{h}, \mathrm{j}, \mathrm{k}$ \\
\hline
\end{tabular}

${ }^{\mathrm{a}}$ Lucatello et al. (2003), ${ }^{\mathrm{b}}$ Ivans et al. (2005), ${ }^{\mathrm{c}}$ Sneden et al. (2003), ${ }^{\mathrm{d}}$ Preston \& Sneden (2001), ${ }^{\mathrm{e}} \mathrm{Hill}$ et al. (2000), ${ }^{\mathrm{f}}$ Barbuy et al. (2005), ${ }^{\mathrm{g}} \mathrm{S}$. Lucatello, R. Gratton, T. Masseron, J. A. Johnson, F. Herwig and S. Tsangarides (unpubl. data), ${ }^{\text {h} A o k i ~ e t ~ a l . ~(2002), ~}{ }^{\mathrm{i} A o k i}$ et al. (2000), ${ }^{\mathrm{j}}$ Norris et al. (1997), ${ }^{\mathrm{k}}$ Tsangarides et al. (2004), ${ }^{\mathrm{l}}$ Sivarani et al. (2006), ${ }^{\mathrm{m}}$ Cohen et al. (2006).

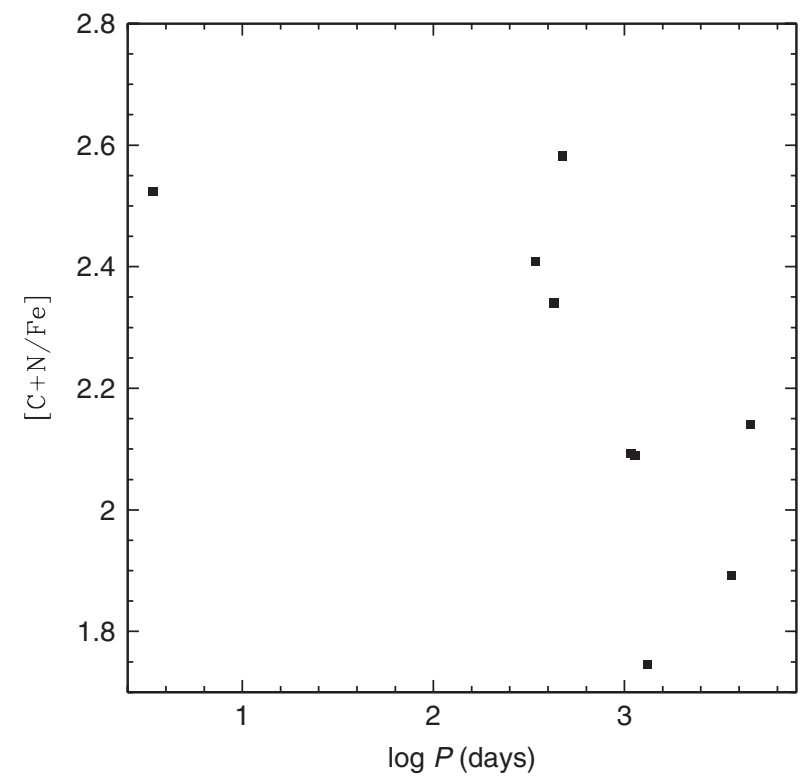

Figure 2 A plot of $[\mathrm{C}+\mathrm{N} / \mathrm{Fe}]$ as a function of orbital period for a sample of 9 CEMP stars.

However, the wind scenario can explain such a relation fully only if we assume that all polluting stars produce roughly the same amount of $\mathrm{C}$ and $\mathrm{N}$, regardless metallicity (note that there is a $[\mathrm{Fe} / \mathrm{H}]$ range of over 1 dex in the stars plotted, see Table 1) and mass. A back of the envelope calculation based on Lugaro et al. (2008) indicates that there is a difference of $\sim 0.5-0.6 \mathrm{dex}$ in $[\mathrm{C}+\mathrm{N} / \mathrm{Fe}]$ produced by a primary of $M=1.5$ and $M=2.2 \mathrm{M}_{\odot}$, assuming a constant metallicity $[\mathrm{Fe} / \mathrm{H}]-2.3$ (note that the full range in $[\mathrm{C}+\mathrm{N} / \mathrm{Fe}]$ in the plot is $\sim 0.9 \mathrm{dex}$ ). If variations in both metallicity and mass are taken into account, the expected spread in $[\mathrm{C}+\mathrm{N} / \mathrm{Fe}]$ is likely much larger. There is currently no theoretical model nor observational evidence that suggest that primary masses and/or initial metallicities of a binary are correlated to orbital periods and semiaxis. It thus seems unlikely that metallicity, initial mass and orbital parameters are tuned to result in the observed correlation.

On the other hand, the issue of mass transfer does not apply at all to the ratios of neutron-capture elements, given

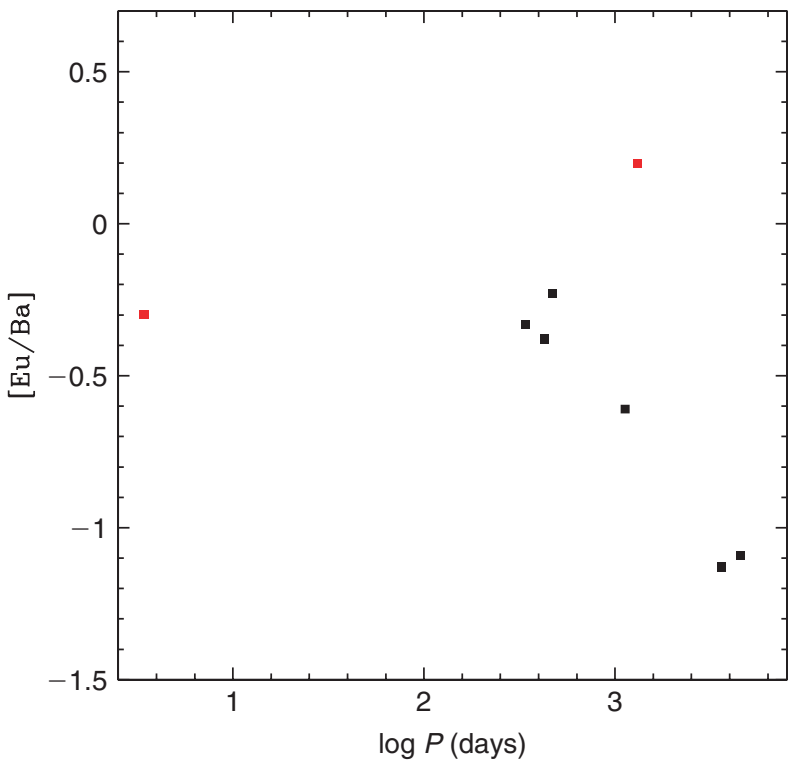

Figure 3 A plot of [Eu/Ba] as a function of period for a sample of 8 stars. Red symbols indicate upper limits in Eu.

that they are all expected to be accreted by the CEMP star. In particular, it is interesting to notice how both the $[\mathrm{Pb} / \mathrm{Ba}]$ and the $[\mathrm{Eu} / \mathrm{Ba}]$ ratios are higher in short period binaries, indicating that they are the object with the most extreme s-patterns, but at the same time the ones for which the $[\mathrm{Eu} / \mathrm{Ba}]$ abundance is too high, according to models, to be accounted for by the $s$-process nucleosynthesis itself.

While the number of stars with well determined period is still too small to draw any solid conclusion, these plots seem to suggest that the membership in a close double system somehow affects the AGB nucleosynthesis, possibly because of mixing enhancement induced by the presence a close companion.

\section{Fluorine in CEMP Stars}

Fluorine is a particularly interesting element, extremely sensitive to the physical conditions within stars. The single stable $\mathrm{F}$ isotope, ${ }^{19} \mathrm{~F}$, is not involved in the main reactions taking place in the cores of stars, however, it can be created and destroyed in several different ways, and its 


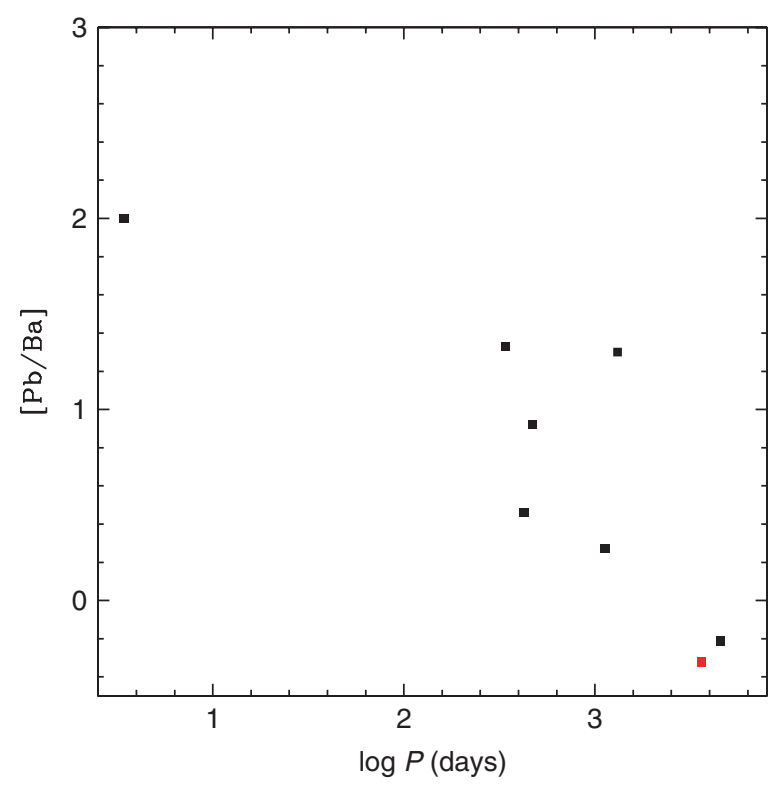

Figure 4 A plot of $[\mathrm{Pb} / \mathrm{Ba}]$ as a function of period for a sample of 8 stars. Red symbols indicate upper limits in $\mathrm{Pb}$ abundance.

dominant source is not clear yet. Theoretical modeling has indicated (at least) three possible sites for F production. Woosley \& Haxton (1988) proposed first that $\mathrm{F}$ is produced in core-collapse supernovae by neutrino spallation on ${ }^{20} \mathrm{Ne} ;{ }^{20} \mathrm{Ne}\left(v, v^{\prime} p\right){ }^{19} \mathrm{~F}$.

On the other hand, modeling shows that lowmetallicity, low-mass $\left(M \leq 3-4 \mathrm{M}_{\odot}\right)$ AGB stars synthesize very large quantities of $\mathrm{F}$ (see Cristallo et al. 2007) via the reaction ${ }^{14} \mathrm{~N}(\alpha, \gamma){ }^{18} \mathrm{~F}\left(\beta^{+}\right){ }^{18} \mathrm{O}(p, \alpha){ }^{15} \mathrm{~N}(\alpha, \gamma){ }^{19} \mathrm{~F}$; where the neutrons are provided by ${ }^{13} \mathrm{C}(\alpha, n){ }^{16} \mathrm{O}$ and the protons mainly by ${ }^{14} \mathrm{~N}(n, p){ }^{14} \mathrm{C}$. Conversely, massive AGB stars are expected to destroy $F$ via Hot Bottom Burning (HBB, see e.g. Smith et al. 2005; Karakas \& Lattanzio 2007).

Finally, Wolf-Rayet stars have been proposed as a possible source for F (Meynet \& Arnould 2000), produced by the same chain of reactions as in the AGB stars. While the precise contribution of each of these three sites to the Galactic F evolution is not known, Renda et al. (2004) showed that the inclusion of all of them in Galactic chemical evolution models is necessary to reproduce the $\mathrm{F}$ abundances measured in Milky Way field stars.

Jorissen, Smith \& Lambert (1992) derived F abundances in giants that recorded the chemical evolution $(\mathrm{K}$ and $\mathrm{M}$ giants), in AGB giants that were dredging up freshly minted $\mathrm{F}$ from their interiors (MS, S, SC, N and J giants) as well as in giants that had been polluted by one of the former (Ba giants), indicating that large amounts of $\mathrm{F}$ are indeed produced in AGB stars.

Schuler et al. (2007) measured for the first time F abundance in a EMP star, deriving for the CEMPs star HE1305+0132 an abundance of $\log \epsilon(\mathrm{F})=+4.96 \pm 0.21$ corresponding to an abundance ratio $[\mathrm{F} / \mathrm{Fe}]=+2.9$. This finding has been discussed and compared to existing nucleosynthesis and mass transfer models in Lugaro et al. (2008), reaching the conclusion that while most CEMPs
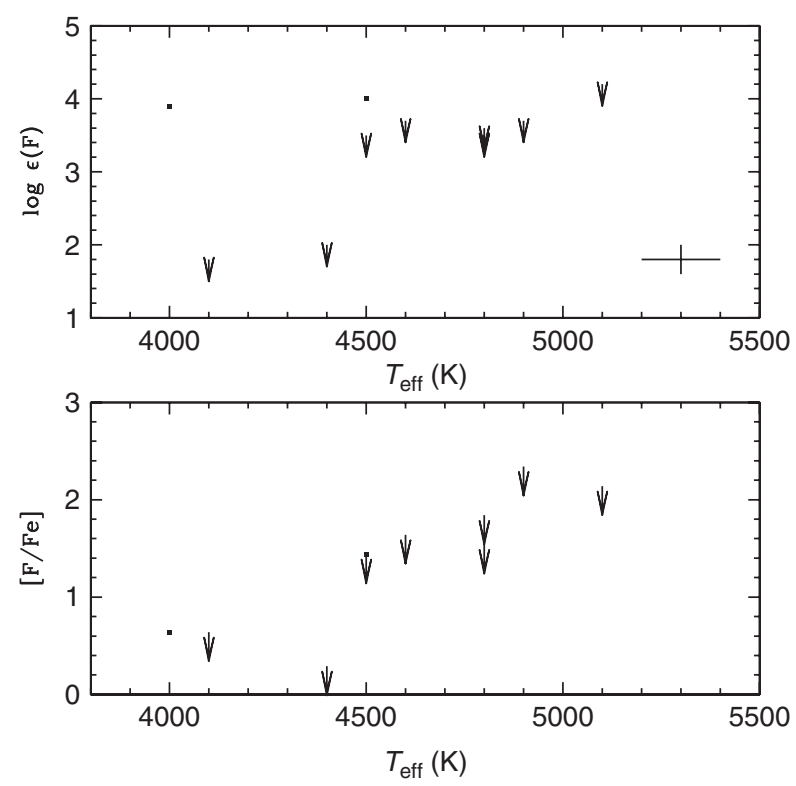

Figure $5 \mathrm{~F}$ abundance as a function of $T_{\text {eff }}$ in 10 CEMP stars. The arrows indicate upper limits, the typical error is indicated in the lower right-hand side.

stars are expected to exhibit a considerable F overabundance, an object with such an extreme F content should be exceedingly rare.

We have obtained $\mathrm{F}$ abundance measurements for two CEMPs $($ at $[\mathrm{Fe} / \mathrm{H}]=-1.3$ and -2 ) and upper limits for eight more CEMP star, including two CEMPno. All the sample stars had published high resolution analyses, providing atmospheric parameters, $\mathrm{Fe}$ and $\mathrm{C}$ abundances. The data were collected with the CRIRES at ESO VLT UT1. The spectra cover the range between 2300 and $2356 \mathrm{~nm}$. The $\mathrm{F}$ abundance was measured from the HF $\mathrm{K}$ line at $2.3 \mu \mathrm{m}$, by spectral synthesis, $\mathrm{N}$ abundance was measured from several $\mathrm{CN}$ lines in the spectral interval under consideration. For more details about the observations, reduction and analyses procedure the interested reader is referred to Lucatello, Johnson \& Masseron (2009).

Given the high sensitivity of HF to temperature, the upper limits levels on the $\mathrm{F}$ abundance are essentially set by the $T_{\text {eff }}$ in the atmosphere of the observed stars (see Figure 5, upper panel). Since F was measured only in two CEMP stars in our sample, both $s$-process rich, and for the remaining eight objects (including the two CEMPno stars) only upper limits could be placed, as far as ${ }^{19} \mathrm{~F}$ is concerned no meaningful comparison is possible between CEMPs and CEMPno stars, and no further piece of information on the formation scenario of CEMPno stars can be derived from the present data. Given the lack of understanding of the nucleosynthetic process to which they owe their peculiar composition, strictly speaking, the inclusion of the upper limits derived for $\mathrm{F}$ abundances in the two CEMPno in the comparison to the AGB models is not appropriate. However, since one of the possible scenarios is indeed that analogous to that invoked for CEMPs stars, we include them in the plots for completeness, clearly marking them. 

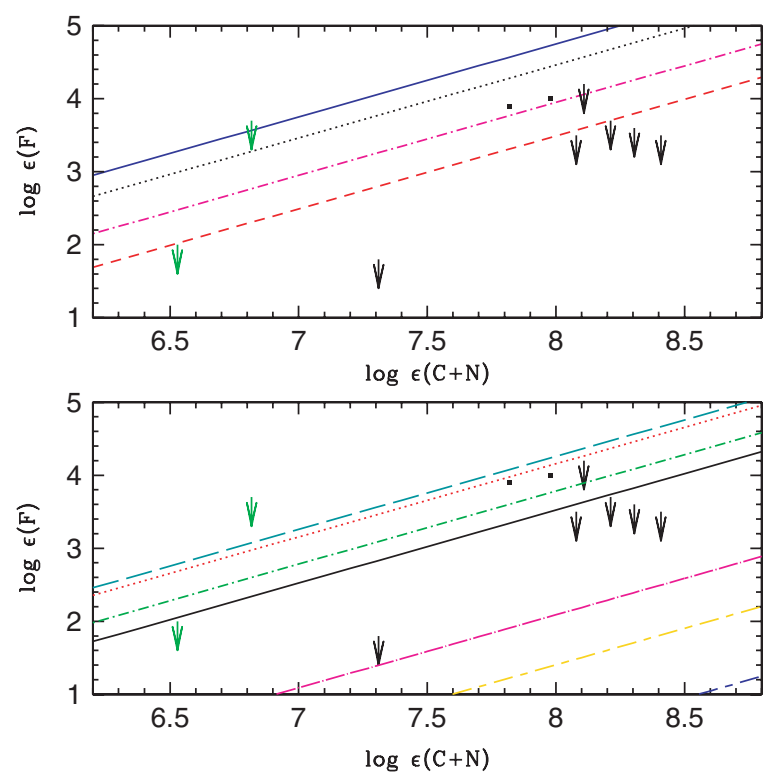

Figure 6 Upper panel: $\mathrm{F}$ abundance as a function of $\mathrm{C}+\mathrm{N}$ in 10 CEMP stars. The arrows indicate upper limits, green symbols are CEMPno stars. Theoretical modeling results are indicated by lines: dotted line indicates the prediction from Karakas \& Lattanzio (2007), the solid line is from Karakas et al. (2008), the dot-dashed line is from Cristallo et al. (2007) and the dashed line is from Stancliffe (2008). All models are for $M=2 \mathrm{M}_{\odot}$ and $[\mathrm{Fe} / \mathrm{H}]=-2.3$. Lower panel: as in upper panel, but plotted models are from Karakas \& Lattanzio (2007) for different masses. Solid black line $1.25 \mathrm{M}_{\odot}$, red dotted line $1.75 \mathrm{M}_{\odot}$, teal dashed line $2.25 \mathrm{M}_{\odot}$, green dot-dashed $2.5 \mathrm{M}_{\odot}$, magenta dot-dashed line $3.0 \mathrm{M}_{\odot}$, short-long dashed yellow line $3.5 \mathrm{M}_{\odot}$ and short-long dashed blue line $4 \mathrm{M}_{\odot}$.

Figure 6 shows the measured $\mathrm{F}$ abundances versus $\mathrm{C}+\mathrm{N}$. The sum of the $\mathrm{C}$ and $\mathrm{N}$ abundance is chosen instead of the individual ones because, as mentioned before, it is likely that some degree of cool bottom processing (currently not included in AGB shell nucleosynthesis models) takes place in the AGB phase burning $\mathrm{C}$ and producing $\mathrm{N}$. Therefore while the prediction of their individual abundances are probably not accurate, their sum is much more robustly predicted. The $\mathrm{F}$ abundances derived show the existence of a scatter (see Figure 5), and this is expected on the basis of the fact that it reflects efficiencies in the mass transfer as well as intrinsic differences in $\mathrm{F}$ production at different metallicities and in different mass primary stars. However the $\mathrm{F}$ to $\mathrm{C}+\mathrm{N}$ ratio is expected to be independent (at least at first approximation) from the amount of mass transfered from the AGB primary to the companion. In fact the bulk of F, C and $\mathrm{N}$ are expected to be accreted in the same way and their abundance ratio to reflect that of AGB nucleosynthesis.

As can be noticed from Figures 5 and 6, no F enhancement to the level published by Schuler et al. (2007) is measured in any of the 10 stars in our sample, with the highest of out measurements at $[\mathrm{F} / \mathrm{Fe}] \simeq 1.9$ and the highest upper limit at $[\mathrm{F} / \mathrm{Fe}] \leq 2.3$, a factor of four smaller than that observed in HE1305+0132 (S. Lucatello, J. Johnson and T. Masseron, unpubl. data). It is important to note that the $\mathrm{Fe}$ abundance in HE1305+0132 adopted in Schuler et al. (2007) is quite uncertain. In fact, given the limited spectral coverage in the Phoenix data, the authors relied on $[\mathrm{Fe} / \mathrm{H}]$ measured from intermediate resolution spectra analysis (Beers et al. 2007), which quoted an $[\mathrm{Fe} / \mathrm{H}]=-2.5 \pm 0.5 \mathrm{dex}$. More recent, high resolution optical data seem however to suggest that HE1305+0132 is in fact more metal rich, with $[\mathrm{Fe} / \mathrm{H}] \simeq-1.92 \mathrm{dex}$ (Schuler et al. 2008). The adoption of such $\mathrm{Fe}$ abundance has the immediate effect of lowering the $[\mathrm{F} / \mathrm{Fe}]$ ratio of $\sim 0.6 \mathrm{dex}$, bringing it to a similar level to those measured in our sample. Moreover, the F abundance itself $\log \epsilon(\mathrm{F})$ would also be affected as the adoption of an appropriate, more metal rich model atmosphere, whose outer layers are cooler than those of the metal poor model atmosphere adopted previously, is expected to result in a lower F abundance. Because of these systematic effects, a direct comparison to our measurements is not possible, and therefore we refrain from including the F measurement for HE1305+0132 in our plots.

Considerable uncertainty exist in the modeling of $\mathrm{F}$ production in AGB stars. In fact, issues such as the inclusion of the a ${ }^{13} \mathrm{C}$ pocket (Lugaro et al. 2004), the appropriate accounting of $\mathrm{CN}$ enhancements in the stellar atmosphere in the opacity calculations (Cristallo et al. 2007), different prescriptions for mass loss and third dredge-up efficiency (Stancliffe 2008), the inclusion of the ${ }^{18} \mathrm{~F}(\alpha, p){ }^{21} \mathrm{Ne}$ reaction and adoption of updated crosssection (Karakas et al. 2008) lead to quite large differences in the predicted $\mathrm{F}$ production.

The upper panel in Figure 6 provides a visual representation of these differences. It shows, along with the data, the predictions from several families of models. The models plotted in the upper panel are all computed for a mass of $2 \mathrm{M}_{\odot}$ and a metallicity of $[\mathrm{Fe} / \mathrm{H}]=-2.3$. The dotted one indicates the prediction from Karakas \& Lattanzio (2007) which is the one used in Lugaro et al. (2008) to account for the F content reported in Schuler et al. (2008). The solid line is from Karakas et al. (2008), integrating the ${ }^{18} \mathrm{~F}(\alpha$, $p)^{21} \mathrm{Ne}$ reaction, adopting the upper limit for its rate (in an effort to try to reproduce the high $\mathrm{F}$ abundances in the Jorissen sample) and including a ${ }^{13} \mathrm{C}$ pocket of $0.002 \mathrm{M}_{\odot}$ at the top layer of the $\mathrm{He}$ - and $\mathrm{C}$-rich inter-shell region. This model is the one among those published in the literature that produces the largest amount of $F$ with respect to $\mathrm{C}$ and $\mathrm{N}$. On the other hand, the dot-dashed line indicates the prediction of the model from Cristallo et al. (2007), whose calculations take into account the appropriate $\mathrm{CN}$ opacities in the stellar evolution model, while the two other models adopt solar scaled composition. Finally, the dashed line shows the prediction from the Stancliffe \& Glebbeek (2008) model with solar scaled composition adopting the prescription of Marigo (2002) for molecular opacities. For the first three models $\mathrm{F}$ production peaks at $\sim 2.2 \mathrm{M}_{\odot}$ (see Lugaro et al. 2008). On the other hand, in Stancliffe (2008) the maximum $[\mathrm{F} / \mathrm{C}+\mathrm{N}]$ ratio is reached at $1.5 \mathrm{M}_{\odot}$.

The predictions for $\mathrm{F}$ productions in $2-\mathrm{M}_{\odot}$ stars cannot fully account for the data. In particular, while the Cristallo et al. (2007) model seem to reproduce the two F abundance 
measurements, it is important to keep in mind that the stars for which actual $\mathrm{F}$ measurements could be obtained have relatively high metallicities of $[\mathrm{Fe} / \mathrm{H}]=-1.3$ and -2.0 , higher than the models. The upper limits are obtained for more metal poor stars, around the $[\mathrm{Fe} / \mathrm{H}] \simeq-2.5$, for which the models have the appropriate metallicity. As mentioned before, for these three models $\mathrm{F}$ production peaks at $\sim 2.2 \mathrm{M}_{\odot}$ (see Lugaro et al. 2008). On the other hand, Stancliffe (2008) prediction comes closer to the data, accounting for both the measurements and one of the upper limits. However, it still cannot account for most of the upper limits derived for CEMPs stars and unlike the other models, the F production peaks at $\sim 1.5 \mathrm{M}_{\odot}$, therefore lower-mass models cannot be invoked to reconcile the discrepancy in this case.

In the lower panel of Figure 6 the predictions for the Karakas \& Lattanzio (2007) models are plotted together with the data. Models are for masses 1.25, 1.75, 2.0, 2.5, 3.0, 3.5 and $4 \mathrm{M}_{\odot}$. The model at $1.75 \mathrm{M}_{\odot}$ seem to reproduce satisfactorily the two $\mathrm{F}$ measurements in our sample, even though it should be kept in mind that, as mentioned, their metallicity is higher than that of the models $([\mathrm{Fe} / \mathrm{H}]=-1.3$ and -2.0$)$. On the other hand, when considering the measurements obtained for the whole sample, it can be noticed that predictions for masses from 1.25 to $2.5 \mathrm{M}_{\odot}$ fail to reproduce the data, with five out of the eight CEMPs stars having their upper limits to the $\mathrm{F}$ to $\mathrm{C}+\mathrm{N}$ ratio lower than computed by the models.

In principle, the models for $3,3.5$ and $4 \mathrm{M}_{\odot}$ could be invoked as the companion polluters to those five CEMPs, as their upper limits $\mathrm{F}$ to $\mathrm{C}+\mathrm{N}$ ratios could be accounted by those models. However, stars of this mass range tend to produce more $\mathrm{N}$ than $\mathrm{C}$, making them essentially $\mathrm{N}$ rich stars rather than $\mathrm{C}$-rich. This not the case of the stars in our sample (four out of those five CEMPs have $[\mathrm{C} / \mathrm{N}]>0)^{2}$. Moreover, a simple back-of-the-envelope calculation, adopting a Salpeter IMF and assuming that (in the considered mass range) the likelihood of having a companion is not affected by mass, indicates that the probability of observing among a sample of eight CEMPs stars with a companion of mass between 1.25 and $4 \mathrm{M}_{\odot}$, five objects that had a companion in the mass range 2.5 to $4 \mathrm{M}_{\odot}$, is basically negligible $\left(10^{-4}\right)$. For these reasons, the possibility that the abundance ratios observed are due to stars more massive than $2.5 \mathrm{M}_{\odot}$ seems unlikely. This suggests that no current model can explain the $\mathrm{F}$ measurements in CEMP stars.

\section{Conclusions}

Our understanding of the range of physical phenomena involved in the CEMP formation processes is still incomplete.

We now know that CEMPs stars are likely the result of AGB mass transfer from an evolved companion, and

\footnotetext{
2 These N-rich, metal poor stars are known to be much less common than expected on the basis of a standard (Salpeter like) initial mass function (IMF) (see Johnson et al. 2007).
}

thus CEMPs stars are the ultimate testing ground for low metallicity AGB modeling, providing crucial constraints for their improvement (e.g. data have shown how some degree of cool bottom processing is probably necessary to explain the observed high $\mathrm{N}$ abundances).

However, several observational results are still poorly understood. As discussed in the previous section, the F abundance ratios measurements obtained in our sample cannot be accounted for with the present set of AGB nucleosynthesis computations. F production is still subject to considerable uncertainties (see Section 4) and perhaps refinement in the modeling guided by an increasing body of observations, will allow to improve our understanding of the production of such elements in AGB stars, and the role of the latter in $\mathrm{F}$ chemical evolution.

On the other hand, we still do not understand why there might be a correlation of some elemental productions and orbital period, such as that shown in Figures 3 and 4.

In spite of considerable effort, no satisfactory explanation has been found for the so-called CEMPr/s stars and the source of their Eu overabundance. The hypotheses put forward so far cannot explain the empirical findings and are challenged either by some feature of the observed abundance patterns or statistical properties of the objects under discussion.

The increase of the number of high resolution CEMP abundance studies can no doubt allow the observers to set even more stringent constraints on their formation schemes. However, the crucial piece of information missing to dramatically improve our understanding of these objects come from radial velocity monitoring. In fact the assessment of the binary fraction and period distributions can distinguish between different scenarios and shed light into the mass transfer mechanisms as well as the nucleosynthesis processes which enriched them (e.g. a SN events taking place in a binaries would decrease their bounding energy, leading to a period distribution shifted toward larger values).

\section{Acknowledgments}

S.L. is grateful to INAF COFIN 2006 and DFG cluster of excellence 'Origin and Structure of the Universe' for partial support. Based in part on observations made with ESO Telescopes at the La Silla and Paranal Observatories under programme IDs 075.D-0120(A), 076.D-0565(A) and 080.D-0606(A).

\section{References}

Aoki, W., Norris, J. E., Ryan, S. G., Beers, T. C. \& Ando, H., 2000, ApJ, 536, L97

Aoki, W., Ryan, S. G., Norris, J. E., Beers, T. C., Ando, H. \& Tsangarides, S., 2002, ApJ, 580, 1149

Aoki, W., Beers, T. C., Christlieb, N., Norris, J. E., Ryan, S. G. \& Tsangarides, S., 2007, ApJ, 655, 492

Barbuy, B., Spite, M., Spite, F., Hill, V., Cayrel, R., Plez, B. \& Petitjean, P., 2005, A\&A, 429, 1031

Barklem, P. S. et al., 2005, A\&A, 439, 129

Beers, T. C. \& Christlieb, N., 2005, ARA\&A, 43, 531 
Beers, T., 2006, Russbach Meeting. Available at http:// www.uni-mainz.de/Organisationen/vistars/talks_russbah2006/ russbah2006_beers.pdf

Beers, T. C., Sivarani, T., Marsteller, B., Lee, Y., Rossi, S. \& Plez, B., 2007, AJ, 133, 1193

Busso, M., Gallino, R., Lambert, D. L., Travaglio, C. \& Smith, V. V., 2001, ApJ, 557, 802

Cohen, J. G. et al., 2006, AJ, 132, 137

Cristallo, S., Straniero, O., Lederer, M. T. \& Aringer, B., 2007, ApJ, 667,489

Frebel, A. et al., 2006, ApJ, 652, 1585

Fujimoto, M. Y., Ikeda, Y. \& Iben, I. J., 2000, ApJ, 529, L25

Gallino, R., Arlandini, C., Busso, M., Lugaro, M., Travaglio, C., Straniero, O., Chieffi, A. \& Limongi, M., 1998, ApJ, 497, 388

Gallino, R., Delaude, D., Husti, L., Cristallo, S., Straniero, O. \& Ryan, S., 2005, NuPhA, 758, 485

Hill, V. et al., 2000, A\&A, 353, 557

Ivans, I. I., Sneden, C., Gallino, R., Cowan, J. J. \& Preston, G. W., 2005, ApJ, 627, L145

Johnson, J. A. \& Bolte, M., 2004, ApJ, 605, 462

Johnson, J. A., Herwig, F., Beers, T. C. \& Christlieb, N., 2007, ApJ, 658,1203

Jorissen, A., Smith, V. V. \& Lambert, D. L., 1992, A\&A, 261, 164

Karakas, A. \& Lattanzio, J. C., 2007, PASA, 24, 103

Karakas, A. I., Lee, H. Y., Lugaro, M., Görres, J. \& Wiescher, M., 2008, ApJ, 676, 1254

Lau, H. H. B., Stancliffe, R. J. \& Tout, C. A., 2008, MNRAS, 385, 301

Lucatello, S., Gratton, R., Cohen, J. G., Beers, T. C., Christlieb, N., Carretta, E. \& Ramírez, S., 2003, AJ, 125, 875

Lucatello, S., Tsangarides, S., Beers, T. C., Carretta, E., Gratton, R. G. \& Ryan, S. G., 2005, ApJ, 625, 825

Lucatello, S., Beers, T. C., Christlieb, N., Barklem, P. S., Rossi, S., Marsteller, B., Sivarani, T. \& Lee, Y. S., 2006, ApJ, 652, L37

Lugaro, M., Ugalde, C., Karakas, A. I., Görres, J., Wiescher, M. Lattanzio, J. C. \& Cannon, R. C., 2004, ApJ, 615, 934
Lugaro, M. et al., 2008, A\&A, 484, L27

Marigo, P., 2002, A\&A, 387, 507

Masseron, T. \& Johnson, J. A., 2008, in International Symposium on Nuclear Astrophysics, Nuclei in the Cosmos, PoS(NIC-X)072

Masseron, T., Johnson, J. A., Plez, B., Van Eck, S., Primas, F., Goriely, S. \& Jorissen, A., 2009, A\&A, submitted (astro$\mathrm{ph} / 0901.4737)$

McClure, R. D., 1984a, PASP, 96, 117

McClure, R. D., 1984b, ApJ, 280, L31

Meynet, G. \& Arnould, M., 2000, A\&A, 355, 176

Norris, J. E., Ryan, S. G. \& Beers, T. C., 1997, ApJ, 488, 350

Newberg, H. J. \& Sloan Digital Sky Survey Collaboration, 2003, BAAS, 35, 1385

Nollett, K. M., Busso, M. \& Wasserburg, G. J., 2003, ApJ, 582, 1036

Preston, G. W. \& Sneden, C., 2001, AJ, 122, 1545

Renda, A. et al., 2004, MNRAS, 354, 575

Ryan, S. G., Aoki, W., Norris, J. E. \& Beers, T. C., 2005, ApJ, 635, 349

Schuler, S. C., Cunha, K., Smith, V. V., Sivarani, T., Beers, T. C. \& Lee, Y. S., 2007, ApJ, 667, L81

Schuler, S., Cunha, K., Smith, V. V., Sivarani, T. \& Beers, T. C., 2008, in International Symposium on Nuclear Astrophysics, Nuclei in the Cosmos, PoS(NIC-X)072

Sivarani, T. et al., 2006, A\&A, 459, 125

Smith, V. V., Cunha, K., Ivans, I. I., Lattanzio, J. C., Campbell, S. \& Hinkle, K. H., 2005, ApJ, 633, 392

Sneden, C. et al., 2003, ApJ, 591, 936

Stancliffe, R. J., Glebbeek, E., Izzard, R. G. \& Pols, O. R., 2007, A\&A, 464, L57

Stancliffe, R. J. \& Glebbeek, E., 2008, MNRAS, 389, 1828

Stancliffe, R. J., 2009, MNRAS, 394, 1051

Tsangarides, S., Ryan, S. G. \& Beers, T. C., 2004, MmSAI, 75, 772

Wanajo, S., 2006, ApJ, 650, L79

Woosley, S. E. \& Haxton, W. C., 1988, Nature, 334, 45

Zhang, B., Ma, K. \& Zhou, G., 2006, ApJ, 642, 1075

Zijlstra, A. A., 2004, MNRAS, 348, L23 